\title{
Computer Modelling the Effects of Chloroquine on KCNJ2 D172N and E299V Mutations-linked Short QT Syndrome
}

\author{
Cunjin Luo ${ }^{1,2}$, Ying $\mathrm{He}^{3}$, Kuanquan $\mathrm{Wang}^{4}$, Henggui Zhang ${ }^{5}$ \\ ${ }^{1}$ School of Computer Science and Electronic Engineering, University of Essex, Colchester, UK \\ ${ }^{2}$ Key Lab of Medical Electrophysiology, Ministry of Education, Institute of Cardiovascular Research, \\ Southwest Medical University, Luzhou, China \\ ${ }^{3}$ School of Computer Science, University of Nottingham, Nottingham, UK \\ ${ }^{4}$ School of Computer Science and Technology, Harbin Institute of Technology, Harbin, China \\ ${ }^{5}$ School of Physics and Astronomy, University of Manchester, Manchester, UK
}

\begin{abstract}
Short QT syndrome (SQTS) is an inherited cardiac disease characterized by ventricular tachyarrhythmias leading to sudden cardiac death (SCD). SQT3 has been linked to the D172N or E299V gain-in-function mutation to Kir2.1, which preferentially increases outward current through channels responsible for inward rectifier $K^{+}$ current $\left(I_{K I}\right)$. Until now, the only effective therapy is implantation of a defibrillator whereas clinical drug therapy is little known about it. Previous studies reported that anti-malarial drug chloroquine was an effective inhibitor of the mutant Kir2.1 channels. The effectiveness of chloroquine has demonstrated in SQT3 associated with D172N mutation, there is a reason to believe that it may be useful in the other mutations in SQT3, such as E299V mutation. Therefore, we aim to predict and compare the potential effects of drug chloroquine on SQT3 D172N and E299V mutations. In this study, the ten Tusscher model of ventricular cell was coupled with the SQT3 mutant $I_{K I}$ patch clamp data. Our simulation data showed that chloroquine prolonged the ventricular cell action potential duration (APD) and $Q T$ interval under SQT3 condition, and demonstrated that chloroquine produced a therapeutic effect on different mutant SQT3. This study provides new evidence that chloroquine may be a potential generic drug for the treatment of SQTS.
\end{abstract}

\section{Introduction}

In 2000, a new cardiac channelopathy was described and called short QT syndrome (SQTS) due to its main feather in the ECG [1]. Currently, more than 400 publications (PubMed: short QT syndrome) have been reported, most of them are clinical cases and investigation of the mechanisms of SQTS. The short QT interval on the
ECG is due to an accelerated cardiac repolarization (and shorter effective refractory period) serving as substrate for atrial and ventricular arrhythmias leading to syncope and even sudden cardiac death (SCD).

Until now, 8 subtypes of SQTS have been described. SQT1, SQT2, and SQT3 are associated with gain-offunction mutant potassium channels, whereas SQT4, SQT5, SQT5 can be caused by loss-of-function mutant calcium channels. SQT7 is associated with a loss-offunction in SCN5A, SQT8 is associated with a mutation in the $\mathrm{CL} / \mathrm{HCO} 3$ exchanger AE3 [2]. Currently, 3 genes encoding potassium channels, KCNH2 (for SQT1), KCNQ1 (for SQT2), KCNJ2 (for SQT3), and 3 genes encoding calcium channels, CACNA1C (for SQT4), CACNB2 (for SQT5), and CACNA2D1 (SQT6) are identified and linked to SQTS [2].

In spite of rapid development in understanding the mechanisms and genetic bases of SQTS, mush less is known about the treatment for SQTS [3, 4]. Due to the high risk of SCD, it has been recommended to implant ICD in SQTS patients. However, ICD is not always feasible for every patient. Therefore, effective drugs are needed for some SQTS patients. Until now, there has been very limited research to test drug effects in SQTS patients. A few anti-arrhythmic drugs such as disopyramide, quinidine, sotalol, ibutilide have been tested in SQT1 patients, but only quinidine has been shown to be effective in the treatment [5].

Recently, anti-malarial drug chloroquine was reported as an effective inhibitor of Kir2.1 channels [6]. Our previous study showed chloroquine prolonged QT interval and terminated reentrant waves in SQT3 [7]. The effectiveness of chloroquine has demonstrated in SQT3 associated with $\mathrm{D} 172 \mathrm{~N}$ mutation, there is a reason to believe that it may be useful in the other mutations in SQT3, such as E299V mutation. Accordingly, in this study, we aim to use computational modelling method to predict and compare the effective effects of chloroquine on SQT3 
associated with D172N and E299V mutations.

\section{Methods}

A mathematical model of the human ventricular cell by ten Tusscher et al. was implemented to simulate the cardiac action potential (AP) [8]. The mathematical formulation for $\mathrm{I}_{\mathrm{K} 1}$ in the original action potential was modified to fit current patch clamp data obtained from Kir2.1 channels (including D172N and E299V mutations). The following equations could reproduce the $\mathrm{I}_{\mathrm{K} 1}$ characteristics in the mutant studies $[9,10]$ :

WT:

$$
\begin{gathered}
\alpha_{\mathrm{K} 1}=\frac{0.07}{1+\mathrm{e}^{0.017\left(V-E_{k}-200.2\right)}} \\
\beta_{\mathrm{K} 1}=\frac{3 \mathrm{e}^{0.0003\left(V-E_{k}+100.2\right)}+e^{0.08\left(V-E_{k}-8.7\right)}}{1+e^{-0.024\left(V-E_{k}\right)}} \\
\mathrm{G}_{\mathrm{K} 1}=4.8 \mathrm{nS} / \mathrm{pF}
\end{gathered}
$$

WT-D172N:

$$
\begin{gathered}
\alpha_{\mathrm{K} 1}=\frac{0.1}{1+\mathrm{e}^{0.023\left(V-E_{k}-199.9\right)}} \\
\beta_{\mathrm{K} 1}=\frac{3 \mathrm{e}^{0.0002\left(V-E_{k}+100.4\right)}+e^{0.07\left(V-E_{k}-9.8\right)}}{1+e^{-0.021\left(V-E_{k}\right)}} \\
\mathrm{G}_{\mathrm{K} 1}=6.27 \mathrm{nS} / \mathrm{pF}
\end{gathered}
$$

WT-E299V:

$$
\mathrm{I}_{\mathrm{K} 1}=\frac{0.11905\left(V-E_{k}+2.4\right)}{0.04092+e^{0.01732\left(V-E_{k}\right)}}-0.36212
$$

Here $E_{\mathrm{K}}$ is the reversal potential for potassium and $V$ is the transmembrane voltage.

Initiation and conduction of action potentials in tissue models was modelled with the mono-domain equation,

$$
C_{m} \frac{\partial V}{\partial t}=-\left(I_{i o n}+I_{s t i m}\right)+\nabla \cdot(D \nabla V)
$$

Where $D$ is the conductivity tensor (diffusion coefficient) describing the tissue conductivity.

For 1D tissue computations, the stand used was a single fibre, $15 \mathrm{~mm}$ long with 100 nodes that were spaced 0.15 $\mathrm{mm}$ apart. The chosen proportion for each region is similar to those used in other studies $[11,12,13]$. The pseudoECG was computed by the method proposed by Gima and Rudy [14].
We analyzed the pharmacological effects of drug/channel binding interactions by using a simple pore block theory. In this study, the fractional block of currents due to chloroquine was modelled by using a blocking factor [7]:

$$
\theta=\frac{1}{1+\frac{\mathrm{IC}_{50}}{[d r u g]^{n H}}}
$$

Where [drug] is the concentration, $\mathrm{IC}_{50}$ is the half-maximal inhibitory concentration, and $\mathrm{nH}$ is Hill coefficient. Chloroquine primarily blocks the $\mathrm{I}_{\mathrm{K} 1}$ and the $\mathrm{I}_{\mathrm{Kr}}$ currents. The resulting ion channel conductivity relative to their original values in the actions of chloroquine are provided in Table 1 [6, 7]. A limitation here is lack of E299V blocking data. We hypothesis that the blocking effect of chloroquine on $\mathrm{I}_{\mathrm{K} 1}$ and $\mathrm{I}_{\mathrm{Kr}}$ under SQT3 D172N and E299V mutant conditions.

Table 1. Block effect of Chloroquine on different currents (\% of original value)

\begin{tabular}{|l|l|l|}
\hline Conditions & Conductivity & $1 \mu \mathrm{M}$ \\
\hline WT & $\mathrm{G}_{\mathrm{K} 1}, \mathrm{G}_{\mathrm{Kr}}$ & $41.6 \%, 38 \%$ \\
\hline WT-D172N & $\mathrm{G}_{\mathrm{K} 1}, \mathrm{G}_{\mathrm{Kr}}$ & $40 \%, 38 \%$ \\
\hline WT-E299V & $\mathrm{G}_{\mathrm{K} 1}, \mathrm{G}_{\mathrm{Kr}}$ & $40 \%, 38 \%$ \\
\hline
\end{tabular}

\section{Results}

\subsection{Single cell simulations}

Figure 1 shows the effects of chloroquine of action potential for the Kir2.1 channels in an EPI ventricular cell. The cell was stimulated at a pacing rate of $1.25 \mathrm{~Hz}$ frequency. The simulation results indicated that chloroquine concentration $1 \mu \mathrm{M}$ was sufficient to prolong action potential both under SQT3 D172N and E299V mutant conditions. Additional simulations on ventricular ENDO and MIDDLE cells (data not shown) showed similar effects of chloroquine to those seen with the ventricular EPI cell action potential. The resting potential values were not markedly changed under both D172N and E299V mutant conditions.

\subsection{Tissue simulations}

Using a strand model of ventricular tissue, we computed a pseudo-ECG (Figure 2). Consistently with action potential simulations, a prolongation of QT interval on the ECG by using chloroquine can be observed both under SQT3 D172N and E299V mutation conditions. 

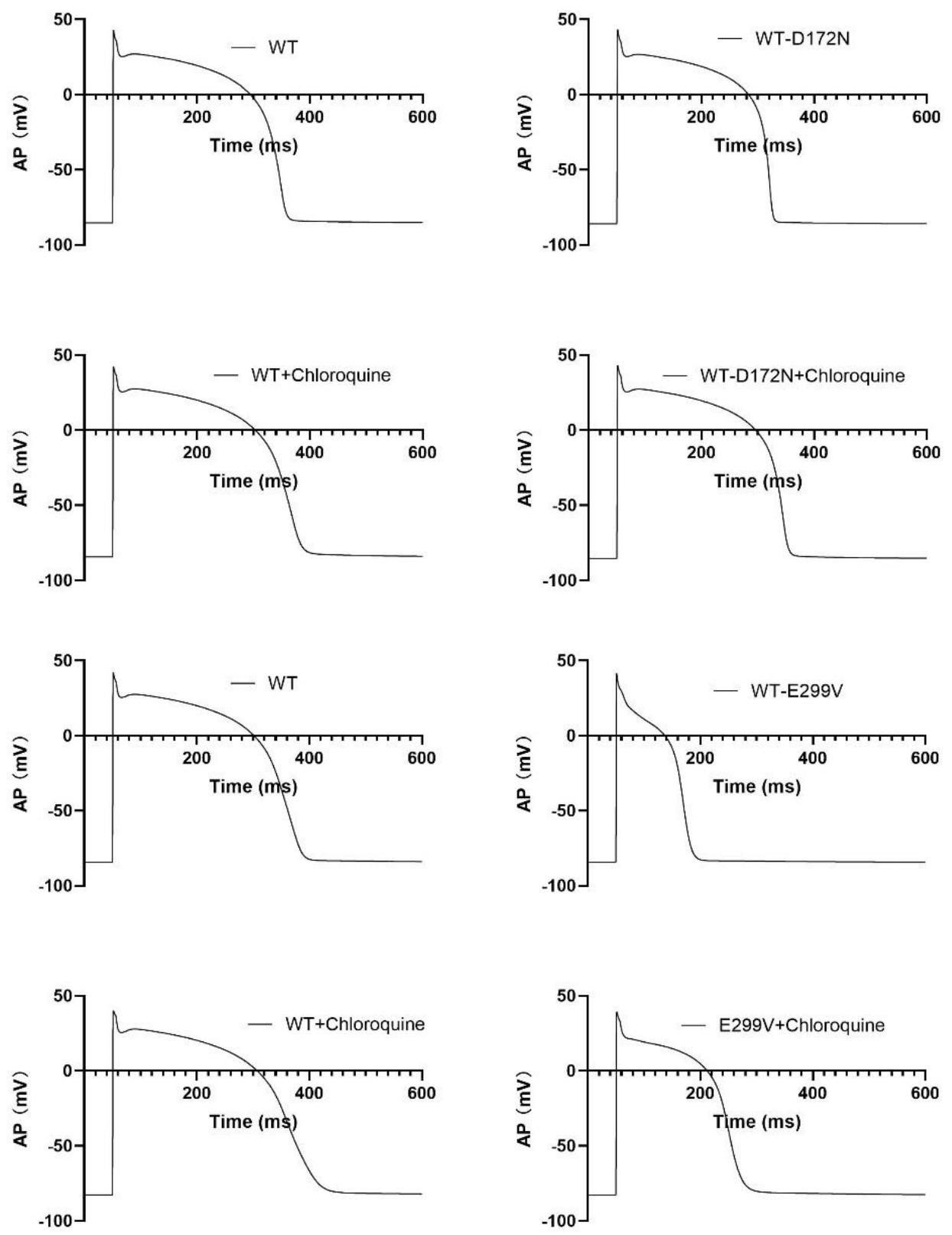

Figure 1. Action potentials under the SQT3 D172N and E299V mutation with the action of Chloroquine

\section{Conclusion}

In the present study, we adopted a computer modelling and simulation approach to gain potential pharmacological effects of chloroquine on SQT3 D172N and E299V mutations. Our findings are summarized as follows: (1) the modified $\mathrm{I}_{\mathrm{K} 1}$ formulations reproduce the dynamic properties of $I_{\mathrm{K} 1}$ under SQT3 D172N and E299V conditions; (2) chloroquine at $1 \mu \mathrm{M}$ concentration prolongs the action potentials; (3) chloroquine prolongs the QT intervals. Collectively, these findings showed that the anti-arrhythmic effects of chloroquine on SQT3 D172N and E299V mutations. This study provides new evidence that chloroquine may be a potential generic drug for the treatment of SQTS.

\section{Acknowledgments}

This work was supported by the National Natural Science Foundation of China (No. 61803318), and Scientific-Technological Collaboration Project under Grant No. 2018LZXNYD-FP02 

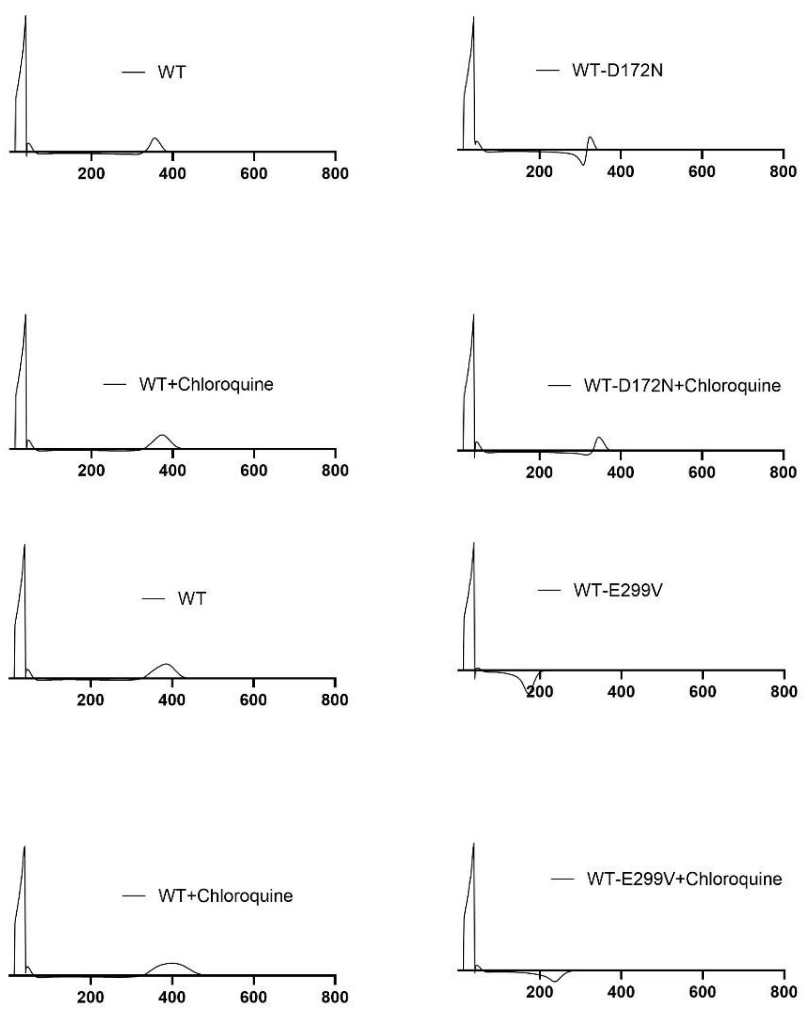

Figure 2. ECGs under the SQT3 D172N and E299V mutation with the action of chloroquine

\section{References}

[1] I Gussak, P Brugada, J Brugada, et al. Idiopathic short QT interval: a new clinical syndrome? Cardiology. 2000; 94: 99-102

[2] I El - Battrawy, J Besler, V Liebe, et al. Long - Term Follow - Up of Patients With Short QT Syndrome: Clinical Profile and Outcome. J Am Heart Assoc. 2018 Dec 4; 7(23): e010073.

[3] C Luo, K Wang, H Zhang. Modelling the effects of quinidine, disopyramide, and E-4031 on short QT syndrome variant 3 in the human ventricles. Physiological measurement 38 (10), 1859.

[4] K Wang, C Luo, Y Yuan, W Lu, H Zhang. Simulation of reentrant wave dynamics in a 2-D sheet of human ventricle with KCNJ2-linked variant 3 short QT syndrome. Computing in Cardiology 2014, 61-64.

[5] I El-Battrawy, J Besler, X Li, et al. Impact of Antiarrhythmic Drugs on the Outcome of Short QT Syndrome. Frontiers in pharmacology, 10 (2019), 771.

[6] A Lopez-Izquierdo, D Ponce-Balbuena, T Ferrer, F Sachse, et al. Chloroquine blocks a mutant Kir2.1 channel responsible for short QT syndrome and normalizes repolarization properties in silico. Cell Physiol Biochem. 2009;24(34):153-60. doi: 10.1159/000233241

[7] C Luo, K Wang and H Zhang. Modelling the effects of chloroquine on KCNJ2-linked short QT syndrome. Oncotarget, 8, 63 (Dec 5 2017), 106511-106526.

[8] ten Tusscher, K. H. and Panfilov, A. V. Alternans and spiral breakup in a human ventricular tissue model. Am J Physiol
Heart Circ Physiol, 291, 3 (Sep 2006), H1088-1100.

[9] I Adeniran, A El Harchi, J Hancox, H Zhang. Proarrhythmia in KCNJ2-linked short QT syndrome: insights from modelling. Cardiovascular Research, Volume 94, Issue 1, 1 April 2012, Pages 66-76.

[10] M Deo, Y Ruan, S Pandit, et al. KCNJ2 mutation in short QT syndrome 3 results in atrial fibrillation and ventricular proarrhythmia. PNAS March 12, 2013110 (11) 4291-4296.

[11] C Luo, K Wang, Y Liu, Y Xia, H Zhang. Effects of quinidine on short QT syndrome variant 2 in the human ventricle: a modelling and simulation study. 2017 Computing in Cardiology (CinC), 1-4.

[12] C Luo, K Wang, M Yuan, et al. Effects of amiodarone on ventricular excitation associated with the KCNJ2-linked short QT syndrome: Insights from a modelling study. 2015 Computing in Cardiology Conference (CinC), 1093-1096.

[13] C Luo, K Wang, H Zhang. Functional effects of islanddistribution of mid-cardiomyocytes on re-entrant excitation waves in the KCNQ1-linked short QT syndrome. 2016 Computing in Cardiology Conference (CinC), 933-936.

[14] K Gima, Y Rudy. Ionic current basis of electrocardiographic waveforms: a model study. Circulation research, 90, 8 (May 3 2002), 889-896.

Correspondence:

Cunjin Luo

Email: cunjin.luo@essex.ac.uk

School of Computer Science and Electronic Engineering

University of Essex

Colchester CO4 3SQ

UK 